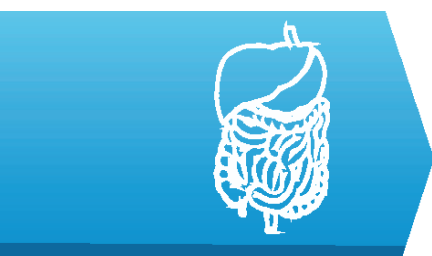

GASTROENTEROLOGY

$2^{\text {nd }}$ Department of Internal Medicine, Iuliu Hatieganu University of Medicine and Pharmacy, Cluj-Napoca, Romania
DOI: $10.15386 / \mathrm{mpr}-1317$

Manuscript received: 27.02.2019

Received in revised form: 02.05.2019

Accepted: 06.05.2019

Address for correspondence:

flaviurusu@yahoo.com

\section{Phenotypic features of patients with post- infectious irritable bowel syndrome}

Flaviu Rusu, Lorena Mocanu, Dan Lucian Dumitraşcu

\begin{abstract}
Background. Although there are many published data in the literature about irritable bowel syndrome (IBS), there are only few data on the long term evolution of patients with post infectious irritable bowel syndrome (PI-IBS) and associated conditions.

Aims. The purpose of our research was to study the evolution of PI-IBS patients in a single tertiary center over a period of four years.

Study Design. Our research was a longitudinal retrospective study.

Methods. We carried out this study based on the survey of the patients records. We recruited two groups of patients: patients with classical IBS and patients with PIIBS. The IBS diagnosis was established using ROME III criteria, which were used at that time. We compared the two groups of patients by analyzing the demographic data, comorbidities, treatment that was prescribed, and evolution after treatment for a period of 48 month on average.
\end{abstract}

Results. From a total of 592 patients that were diagnosed with IBS between 20132016, we identified a subgroup of 64 patients with PI-IBS. These patients were also divided into two subgroups, depending on the main symptoms, 51 with PI-IBS diarrhea and 13 of them with PI-IBS-constipation. IBS is the most commonly diagnosed among women, 45 patients were women and 15 were men. Regarding the frequency of occurrence of a certain subtype by gender, no significant differences were observed in both IBS and PI-IBS. We noticed a higher incidence of patients residents of an urban community in both groups.

The main symptoms were: abdominal pain and bowel disorders (constipation, diarrhea). There was a significant difference between the two groups regarding the onset of the symptoms. From the total of 64 patients with PI-IBS, $88.3 \%$ presented a sudden onset of symptoms (mainly abdominal pain) unlike the other group where $81 \%$ of them presented a progressive onset, with an insidious progress and sporadic exacerbation. Most patients (65.63\%) presented an improvement after the treatment, $25.56 \%$ oscillating periods but with a significant decrease of intensity of symptoms, and $7.8 \%$ did not show improvement after treatment.

The most common associated diseases were depression and anxiety (34.37\%).

Conclusion. IBS is generally present in approximately $11 \%$ of the population, with PI-IBS patients accounting for approximately $10 \%$ of them. Female gender is more common in both IBS and PI-IBS. Patients with PI-IBS are a burden in the health system in terms of the important economic resources used for diagnosis and treatment.

Keywords: IBS, PI-IBS, symptoms, treatment, associated diseases

\section{Introduction}

IBS is a common disorder, the mean prevalence among individual countries ranges from $1.1 \%$ in France and Iran to $35.5 \%$ in Mexico [1]. The underlying pathophysiology and the clinical presentation of IBS are extremely diverse [2]. PI-IBS has recently been considered as a separate entity and includes abdominal discomfort, bloating and flatulence. Unlike IBS, PI-IBS has a well-defined onset [3]. Published data estimate that approximately $10 \%$ of cases of IBS are PI-IBS subtype [4]. Most patients with PI-IBS developed IBS-D (63\%) and only a small part developed IBS-C $(13 \%)$, the rest of the patients showing an alternation of symptoms (24\%) [5]. Acute infectious gastroenterocolitis is the main incriminated disease in PI-IBS, between 7 and $31 \%$ of patients developing PI-IBS 
over time [6]. The most common etiological agents identified were Salmonella, Shigella and Campylobacter [7]. The main risk factors associated with the occurrence of PI-IBS after an episode of gastroenterocolitis are represented by female gender, old age, a long-lasting and severe infectious episode, antibiotic use and concomitant psychiatric disorder [8-11]. The preexistence of a psychological condition (depression, anxiety) has been associated with an increased prevalence of PI-IBS development [12]. A big importance was given to the persistence of inflammation in the intestinal mucosa after the acute infectious process [13]. Recent studies have investigated the role of granins and toll-like receptors as markers of inflammation in IBS [14]. The intestinal microbiota of each individual depends on several factors: genetic, diet, age, antibiotic use, intestinal infections [15]. Subjects with IBS have a bacterial excessive overcrowding in the small intestine (SIBO) [16]. The purpose of our research was to study the evolution of PI-IBS patients in a single tertiary center over a period of four years.

\section{Methods \\ Protocol}

We performed a retrospective longitudinal study using data from our Medical Database, from Second Medical Department, Cluj-Napoca. Patients recruitment was performed over a 48-month period (2013-2016). We recruited two groups of patients: patients with PI-IBS and patients with classical IBS (non PI-IBS) used as controls. The IBS diagnosis was established using ROME III criteria, which were standard at that time. We compared the two groups of patients by analyzing the demographic data, comorbidities, treatment that was prescribed and evolution after treatment for a period of 48 months on average. Given the time of diagnosis, some patients were observed over a longer period of time than patients who were later entered into the clinic database.

The clinical reassessment of the patients was performed according to the clinic protocol, initially once a month and then once every six months. At each visit the patients were assessed for major symptoms such as abdominal pain or discomfort, bowel disorders, flatulence and bloating. Their evolution and the response to treatment was also noted.

Inclusion criteria: only new patients (over 18 years old) who were not previously diagnosed with IBS or PIIBS were included in the study so that the first diagnosis was established in our clinic.

Exclusion criteria: pregnant women, oncological patients and patients with alcohol dependence. These exclusion criteria were selected due to the IBS-like symptoms that may be experienced by pregnant women and patients with some cancers such as colorectal cancer. We excluded the patients with alcohol dependence because of the non-compliance shown by these patients, especially regarding the hygienic-dietary regime, but also regarding the correct administration of the prescribed treatment.
Subjects: after a careful review of the inclusion and exclusion criteria, we identified 592 eligible patients for our study.

Among the studied parameters, the most important were: age, gender, onset of symptoms, the main associated illnesses, the environment of origin (urban or rural), the treatment and the evolution over time.

\section{Statistical analysis}

We recruited two groups of patients: patients with classical IBS and patients with PI-IBS. These patients were also divided into two subgroups, depending on the main symptoms, 51 with PI-IBS - diarrhoea and 13 of them with PI-IBS-constipation. We compared the two groups of patients by analyzing the demographic data, comorbidities, treatment that was prescribed, and evolution after treatment for a period of 48 month in average.

The statistical method that we used was the student $t$ test to determine the probability of difference between the classical IBS group and the PI-IBS with the two subgroups: PI-IBS - diarrhoea and PI-IBS-constipation. To do this, we used the Windows 10 operating system. The $t$ score is a ratio between the difference between two groups and the difference within the groups. The parameters analyzed were previously described. The significance of the results was interpreted using the $t$ test and also the Pearson's chisquared test. We used the chi-squared statistic to calculate a p-value by comparing the value of the statistic to a chisquared distribution. Every t-value has a p-value to go with it. A p-value is the probability that the results from the sample data occurred by chance. Low p-values are good; tThey indicate that the data did not occur by chance. Only values of the $\mathrm{p}$ index of $<0.05$ were considered significant.

\section{Ethical issues}

In order to perform this study, we had the approval of the local ethics committee.

\section{Results}

From the total of 592 identified subjects, 64 were with PI-IBS and the IBS control group counted 528 subjects. The age range was $19-85$ years for the PI-IBS group, with an average of 43.4 years. For the control group the range was between 18 and 87 years, with an average of 41.7 years (Table I).

IBS is more commonly diagnosed in females, and this can be highlighted among patients at the 2-nd Medical Clinic, both in IBS and PI-IBS. From a total of 528 patients with IBS, only 174 were men, most of the group being women. The same was observed in the SII-PI group in which from a total of 64 patients, 45 were women and only 15 were men. There was no statistically significant difference between the male / female ratio in the two groups of patients (chi-square: $2.77, \mathrm{p}=0.87$ )

We noticed a higher incidence of urban patients, both for patients with classical IBS and for patients with PI-IBS, but statistically insignificant (chi-square test: $2.10, \mathrm{p}=0.34$ ). 
We identified a statistical difference between the classic IBS patients and the PI-IBS patients regarding the subtypes (Table II). In the PI-IBS group prevailed the PIIBS-D (51 patients $-80 \%$ ) to the detriment of PI-IBS-C subtype (13 patients - 20\%), and in the classic IBS prevailed the IBS-C group (48\%) (chi-square: $21.4177, \mathrm{p}=0.000022$ ). There was a statistically significant difference between the two constipation groups (chi-square: $11.976, \mathrm{p}=0.000539$ ) and between the two diarrhea groups (chi-square: 5.795, $\mathrm{p}=0.016072$ ).

There was a statistically insignificant difference between the two groups of women with predominance of diarrhea (chi-square: $0.0091, \mathrm{p}=0.923$ ) and a statistically significant difference between the two groups of females with IBS and PI-IBSI with the predominance of constipation (chi-square: 14.1708, $\mathrm{p}=0.000167$ ). There was a statistically insignificant difference between the two groups of men with diarrhea predominance (chi-square: $0.027, \mathrm{p}=0.869$ ) but there is a statistically significant difference between the two groups of males with IBS and PI-IBSI with the predominance of constipation (chi-square: 8.6886, $\mathrm{p}=0.0032$ ).

The most common symptoms observed in these patients (Table III, IV) were: impaired bowel function and abdominal pain. Other symptoms that have been encountered in fewer patients were flatulence and bloating.

Table I. Demographic data

\begin{tabular}{l|c|c|l|c|c}
\hline IBS & No. & $\%$ & PI-IBS & No. & $\%$ \\
\hline Gender & 528 & 100 & Gender & 64 & 100 \\
Male & 174 & 33 & Male & 19 & 29.68 \\
Female & 354 & 67 & Female & 45 & 70.32 \\
Age & 528 & 100 & Age & 64 & 100 \\
$18-30$ & 206 & 39 & $19-30$ & 22 & 34.37 \\
$31-50$ & 269 & 51 & $31-50$ & 28 & 43.75 \\
$51-87$ & 53 & 10 & $51-85$ & 14 & 21.88 \\
Rural & 167 & 32 & Rural & 26 & 41 \\
Male & 60 & 35.92 & Male & 8 & 30.77 \\
Female & 107 & 64.08 & Female & 18 & 69.23 \\
Urban & 361 & 68 & Urban & 38 & 59 \\
Male & 120 & 33.24 & Male & 17 & 44.73 \\
Female & 241 & 66.76 & Female & 21 & 55.27
\end{tabular}

Table II. IBS and PI-IBS subtypes

\begin{tabular}{|l|r|c|c|c|}
\hline IBS-C & IBS-D & IBS-M & PI-IBS-D & PI-IBS-C \\
\hline $48 \%$ & $46 \%$ & $6 \%$ & $80 \%$ & $20 \%$ \\
\hline
\end{tabular}

Table III. Frequency of symptoms in IBS patients.

\begin{tabular}{|l|c|c|c|c|}
\multirow{2}{*}{\multicolumn{1}{c}{ Frequency }} & \multicolumn{4}{|c|}{ IBS symptoms (\%) } \\
\cline { 2 - 5 } & Abdominal pain & Bowel disorders & Bloating & Flatulence \\
\hline Daily & 18.2 & 33.1 & 17.6 & 25.7 \\
At least 2-3 times/week & 55.6 & 58.3 & 71.8 & 61.4 \\
1-4 episodes/month & 26.2 & 8.6 & 10.6 & 12.9
\end{tabular}

Table IV. Frequency of symptoms in PI-IBS patients.

\begin{tabular}{|l|c|c|c|c|}
\multirow{2}{*}{\multicolumn{1}{c|}{ Frequency }} & \multicolumn{4}{|c|}{ PI-IBS symptoms (\%) } \\
\cline { 2 - 5 } & Abdominal pain & Bowel disorders & Bloating & Flatulence \\
\hline Daily & 45.2 & 68.8 & 33.6 & 26.3 \\
At least 2-3 times/week & 38.1 & 23.5 & 49.7 & 58.2 \\
1-4 episodes/month & 16.7 & 7.7 & 16.7 & 15.5
\end{tabular}


There were significant differences between patients who had spontaneously developed IBS, as opposed to patients with IBS emerging after an infectious process (chi-square: 128.8288, $\mathrm{p}<0.00001$ ). From the total of 64 patients with PI-IBS, $88.3 \%$ had an acute onset, mainly abdominal pain. The other symptoms were impaired bowel, bloating and flatulence, and out of a total of 528 patients with spontaneous IBS, without being preceded by an acute gastrointestinal infection, only $21 \%$ had a sudden onset of symptoms, most of them having a progressive and insidious onset of symptoms, with episodes of sporadic complaints of different intensities (79\%) (Table V).

Out of a total of 64 PI-IBS patients, $65.6 \%$ responded favorably to the therapy, with a significant reduction in the incidence of symptomatology. $26.5 \%$ had oscillating periods but a decrease in the intensity of the symptoms was noted. $7.8 \%$ of patients did not show significant improvements after the therapy (Table VI).

The main classes of medication used in our country regarding the treatment of IBS and PI-IBS are: spasmolytics, anticholinergics, opiate agonists, calcium antagonists, serotoninergic antagonists and antidepressants, antibiotic (rifaximin), laxatives, prokinetics and prostaglandin derivatives, diosmectite, bile acids chelators and opiate agonists, dimeticona, simeticona and activated charcoal. Depending on the severity and type of symptoms, it was decided to administer the treatment either as monotherapy or in combination. The intensity of symptoms was quantified both before and after the treatment. There was a significant decrease in symptom severity, in approximately $30 \%$ of patients with PI-IBS-C and in approximately $50 \%$ of patients with PI-IBS-D, following drug therapy. There was a statistically significant difference between the two groups regarding the evolution of symptoms after treatment (chi-square: 20.1181, $\mathrm{p}=0.00016$ ).

The treatment available includes drug therapy as well as non-medical treatment like cognitive behavioural therapy. We mention that in carefully selected cases, recommendations for psychiatric or psychological consultation were made at discharge or at regular checkups but we did not have enough data collected from patients to interpret them except for the diagnoses established by the psychiatrist or by the psychologist.

Patients with PI-IBS have also frequently experienced other symptoms than those specific to this disorder, among which the most common were: headache, migraine, anxiety, depression, nausea, insomnia, eructation and palpitations (Table VII).

Depression and anxiety are quite commonly found in these patients. From the total of 64 patients with PI-IBS, 22 of them $(34.37 \%)$ have been diagnosed with depression or anxiety (Table VIII). There was a statistically insignificant difference between the two groups regarding the incidence of depression (chi-square: $0.8447, \mathrm{p}=0.655$ ).

Table V. The onset of symptoms.

\begin{tabular}{|l|c|c|}
\hline The onset of symptoms & Sudden onset & Insidious onset \\
\hline IBS & $21.2 \%$ & $78.8 \%$ \\
\hline PI-IBS & $89 \%$ & $11 \%$
\end{tabular}

Table VI. Evolution of patients after treatment.

\begin{tabular}{l|c|c|c|}
\hline Evolution & $\begin{array}{l}\text { with a decrease in the } \\
\text { frequency of symptoms }\end{array}$ & $\begin{array}{l}\text { fluctuating evolution but with } \\
\text { decreasing symptom severity }\end{array}$ & $\begin{array}{l}\text { without favorable } \\
\text { evolution }\end{array}$ \\
\hline IBS & $37.1 \%$ & $40.9 \%$ & $22 \%$ \\
\hline PI-IBS & $65.6 \%$ & $26.5 \%$ & $7.8 \%$
\end{tabular}

Table VII. Other common symptoms in IBS patients (no. and \%).

\begin{tabular}{c|c|c|c|c|c|c|c} 
Symptoms & Headache & Migraine & Anxiety & Nausea & Insomnia & Eructation & Palpitations \\
\hline \multirow{2}{*}{ IBS } & $38 \%$ & $30.6 \%$ & $22.9 \%$ & 20.4 & $16.2 \%$ & $11.74 \%$ & $6.81 \%$ \\
& 201 & 162 & 121 & 108 & 86 & 62 & 36 \\
\multirow{2}{*}{ PI-IBS } & $32.8 \%$ & $25 \%$ & 20.3 & $15.6 \%$ & $12.5 \%$ & $9.3 \%$ & $7.8 \%$ \\
& 21 & 16 & 13 & 10 & 8 & 6 & 5
\end{tabular}

Table VIII. The incidence of depression in patients with PI-IBS and IBS.

\begin{tabular}{l|c|c} 
Depression/anxiety & Patients with depression/anxiety & Patients without depression/anxiety \\
\hline IBS & $28.03 \%$ & $71.97 \%$ \\
PI-IBS & $34.37 \%$ & $65.63 \%$
\end{tabular}


Table IX. Associated conditions PI-IBS.

\begin{tabular}{|l|c|c|c|c|c|}
\hline Associated conditions & Depression & Hemorrhoidal disease & Fibromyalgia & Colon polyps & Diverticulosis \\
\hline No. & 22 & 18 & 13 & 10 & 9 \\
\hline$\%$ & 34.37 & 28.12 & 20.31 & 15.62 & 14.06 \\
\hline
\end{tabular}

Table X. Associated conditions IBS.

\begin{tabular}{|l|c|c|c|c|c|}
\hline Associated conditions & Depression & Dyslipidemia & Hemorrhoidal disease & Fibromyalgia & Obesity \\
\hline No. & 121 & 139 & 107 & 103 & 99 \\
\hline$\%$ & 22.9 & 26.32 & 20.26 & 19.5 & 18.75 \\
\hline
\end{tabular}

An important number of patients had multiple associated diseases, among which the most often noted are: depression, hemorrhoidal disease, fibromyalgia, colon polyps and diverticulitis, diabetes mellitus, chronic gastritis and hypothyroidism (Table IX).

Patients with IBS may have also multiple associated conditions (Table X).

\section{Discussion}

The pathogenesis of PI-IBS is the subject of recent studies, indicating the involvement of multiple pathogenetic factors. Acute infectious gastroenterocolitis is the main incriminated disease in PI-IBS and the most common etiological agents identified were Salmonella, Shigella and Campylobacter [17].

Similar to IBS, the preexistence of a psychological condition was associated with an increased prevalence of PI-IBS development [12]. The prevalence of depression and anxiety is considerably lower among patients who develop PI-IBS than in patients with classic IBS (26\% vs 54\%) [18].

The results of a study published by Spiller et al. revealed the presence of inflammation in the intestinal mucosa by performing colonic biopsies at 2,6 and 52 weeks after a gastroenterocolitis with Campylobacter jejuni, with numerous $\mathrm{T}$ lymphocytes and calprotectin-positive macrophages [7]. Chavez et al presented the role of nitric oxide production in increasing intestinal permeability mediated by cytokines [19]. Motility and intestinal secretion is also influenced by the amount of serotonin released by enterochromaffin cells [20]. Fatty acid binding protein (FABP) is involved in the transport of fatty acids from the lumen into the enterocytes. It has been shown that there is a link between this protein and intestinal mucosal lesions found in inflammatory diseases [21]. It is released into circulation when integrity of the enterocyte membrane is impaired, being also a possible predictor of intestinal ischemia [22].

Wang et al. presented in a study on 89 patients a comparison between classical IBS and PI-IBS. Significantly higher levels of CRP and FABP were seen in serum of PIIBS patients compared to patients with noninfectious IBS. $70.8 \%$ of subjects with PI-IBS had diarrhea as the primary symptom, and the anxiety score was significantly higher in PI-IBS patients [23].
A study on 50 subjects analyzed the microbiota in the stool. Subjects with IBS showed a lower level of Bifidobacterium and a higher level of Enterobacteriacea in the stool compared to healthy subjects [24]. Another study revealed a high number of Proteobacteria and Firmicutes in the stool among patients with IBS with predominance of diarrhea and a low number of Actinobacteria and Bacteroidetes compared to healthy subjects [25]. Food intolerance has also been investigated as a possible cause of symptomatology in patients with PI-IBS. Bacterial overpopulation of the small intestine is currently the subject of several studies that aim to investigate its role as a potential etiologic factor of PI-IBS [26-28].

To our knowledge, this is the first study to report the results of a 4-year follow-up of a group of patients with PIIBS in Romania. The data published so far on PI-IBS is not numerous and it is known that this disorder definitely affects the patients' quality of life. We conducted this study aiming at highlighting the main phenotypic particularities of patients with PI-IBS registered in our clinic, the patients being evaluated retrospectively during a period of four years. We investigated the demographic data, symptoms, concomitant diseases, treatment and the response to therapy among patients with PI-IBS. The treatment given to these patients has the role of reducing inflammation in the gut, improving intestinal barrier function and reducing visceral sensitivity [29-30]. Drug treatment administered in combination conducted to positive results, with a better evolution of patients compared with the administration in monotherapy. Administration of anxiolytics in PI-IBS patients with associated anxiety and depression resulted in a favorable clinical evolution.

The results of our study showed new data, some comparable to those described in literature, especially in patients from Europe, and others different from the literature data.

From an epidemiological point of view, IBS is found met globally in up to $35.5 \%$ of patients with gastrointestinal disorders [1], those with PI-IBS accounting for about 10-11\% of them. The prevalence of this syndrome varies by region, gender, geographical position and economic conditions. The results of studies published so far have shown a higher prevalence among young people and adults, especially female subjects, with a slightly increased predisposition for 
urban patients. In our study, approximately $20 \%$ of patients with gastrointestinal disorders were diagnosed with IBS, of whom $11 \%$ were diagnosed with PI-IBS.

It is very important to establish the correct diagnosis of PI-IBS. This should be considered in patients with no prior symptom-specific IBS (according to Rome IV criteria), with an acute onset of an acute gastroeterocolitis episode. It is mandatory to perform a differential diagnosis with organic substrate diseases. We performed multiple investigations in our patients with PI-IBS that were supplemented with inferior digestive endoscopy in order to exclude an organic substrate.

Study limits were mainly related to the relatively small number of PI-IBS patients, but also that some investigations were not made due to financial problems (the determination of serum or intestinal mucosal level of proinflammatory cytokines, the bacterial overgrowth test).

\section{Conclusions}

IBS is generally present in approximately $11 \%$ of the population, with PI-IBS patients accounting for approximately $10 \%$ of them. Female gender is more common in both IBS and PI-IBS. We noticed a higher incidence of urban patients. We identified a statistical difference between the classic IBS patients and the PI-IBS patients regarding the subtypes. There was a statistically significant difference between the two constipation groups. There were significant differences between patients who had spontaneously developed IBS, as opposed to patients with IBS emerging after an infectious process. Out of a total of 64 PI-IBS patients, $65.6 \%$ responded favorably to the therapy, with a significant reduction in the incidence of symptomatology. There was a statistically significant difference between the two groups regarding the evolution of symptoms after treatment. Depression and anxiety are quite commonly found in these patients. Patients with PI-IBS are a burden in the health system in terms of the important economic resources used for diagnosis and treatment.

\section{References}

1. Sperber AD, Dumitrascu D, Fukudo S, Gerson C, Ghoshal UC, Gwee KA, et al. The global prevalence of IBS in adults remains elusive due to the heterogeneity of studies: a Rome Foundation working team literature review. Gut. 2017;66:1075-1082.

2. Boeckxstaens GE, Drug V, Dumitrascu D, Farmer AD, Hammer J, Hausken T, et al. Phenotyping of subjects for large scale studies on patients with IBS. Neurogastroenterol Motil. 2016;28:1134-1147.

3. Drossman DA. The functional gastrointestinal disorders and the Rome III process. Gastroenterology. 2006;130:13771390.

4. Klem F, Wadhwa A, Prokop LJ, Sundt WJ, Farrugia G, Camilleri M, et al. Prevalence, Risk Factors, and Outcomes of Irritable Bowel Syndrome After Infectious Enteritis: A Systematic Review and Meta-analysis. Gastroenterology. 2017;152:1042-1054.e1.

5. Spiller R, Garsed K. Postinfectious irritable bowel syndrome. Gastroenterology. 2009;136:1979-1988.

6. Barbara G, Cremon C, Pallotti F, De Giorgio R, Stanghellini V, Corinaldesi R. Postinfectious irritable bowel syndrome. J Pediatr Gastroenterol Nutr. 2009;48 Suppl 2:S95-S97.

7. Spiller RC. Postinfectious irritable bowel syndrome. Gastroenterology 2003;124:1662-1671.

8. Neal KR, Hebden J, Spiller R. Prevalence of gastrointestinal symptoms six months after bacterial gastroenteritis and risk factors for development of the irritable bowel syndrome: postal survey of patients. BMJ. 1997;314:779-782.

9. Connor BA. Sequelae of traveler's diarrhea: focus on postinfectious irritable bowel syndrome. Clin Infect Dis. 2005; 41 Suppl 8:S577-S586.

10. Wadhwa A, Al Nahhas MF, Dierkhising RA, Patel R, Kashyap P, Pardi DS, et al. High risk of post-infectious irritable bowel syndrome in patients with Clostridium difficile infection . Aliment Pharmacol Ther. 2016;44:576-582.

11. Barbara G, Stanghellini V, Berti-Ceroni C, De Giorgio R, Salvioli B, Corradi F, et al. Role of antibiotic therapy on long-term germ excretion in faeces and digestive symptoms after Salmonella infection. Aliment Pharmacol Ther. 2000;14:1127-1131.

12. Dunlop SP, Jenkins D, Neal KR, Spiller RC. Relative importance of enterochromaffin cell hyperplasia, anxiety, and depression in postinfectious IBS. Gastroenterology. 2003;125:1651-1659.

13. Barbara G, De Giorgio R, Stanghellini V, Cremon C, Salvioli B, Corinaldesi R. New pathophysiological mechanism in irritable bowel syndrome. Aliment Pharmacol Ther. 2004;20 Suppl 2:1-9.

14. Rusu F, Dumitrașcu DL. Four years follow-up of patients with irritable bowel syndrome. Rom J Intern Med. 2015;53:65-74.

15. Spiller R, Lam C. An Update on Post-infectious Irritable Bowel Syndrome: Role of Genetics, Immune Activation, Serotonin and Altered Microbiome. J Neurogastroenterol Motil. 2012;18:258-268.

16. Pyleris E, Giamarellos-Bourboulis EJ, Tzivras D, Koussoulas V, Barbatzas C, Pimentel M. The prevalence of overgrowth by aerobic bacteria in the small intestine by small bowel culture: relationship with irritable bowel syndrome. Dig Dis Sci. 2012;57:1321-1329.

17. Andresen V, Löwe B, Broicher W, Riegel B, Fraedrich $\mathrm{K}$, von Wulffen $\mathrm{M}$, et al. Post-infectious irritable bowel syndrome (PI-IBS) after infection with Shiga-like toxinproducing Escherichia coli (STEC) O104:H4: A cohort study with prospective follow-up. United European Gastroenterol J. 2016;4:121-131.

18. DuPont AW. Postinfectious irritable bowel syndrome. Clin Infect Dis. 2008;46:594-599.

19. Chavez AM, Menconi MJ, Hodin RA, Fink MP. Cytokineinduced intestinal epithelial hyperpermeability: role of nitric oxide. Crit Care Med. 1999;27:2246-2251.

20. Coates MD, Mahoney CR, Linden DR, Sampson JE, Chen J, Blaszyk H, Crowell MD, et al. Molecular defects in mucosal serotonin content and decreased serotonin reuptake 
transporter in ulcerative colitis and irritable bowel syndrome. Gastroenterology. 2004;126:1657-1664.

21. Gregory KE, Winston AB, Yamamoto HS, Dawood HY, Fashemi T, Fichorova RN, et al. Urinary intestinal fatty acid binding protein predicts necrotizing enterocolitis. J Pediatr. 2014;164:1486-1488.

22. Kittaka H, Akimoto H, Takeshita H, Funaoka H, Hazui H, Okamoto M, et al. Usefulness of intestinal fatty acid-binding protein in predicting strangulated small bowel obstruction. PLoS One. 2014;9:e99915.

23. Wang J, Lu S, Zhao S. Post-infectious and non post-infectious irritable bowel syndrome: A comparative study. Pak J Med Sci. 2016;32:116-119.

24. Si JM, Yu YC, Fan YJ, Chen SJ. Intestinal microecology and quality of life in irritable bowel syndrome patients. World J Gastroenterol. 2004;10:1802-1805.

25. Krogius-Kurikka L, Lyra A, Malinen E, Aarnikunnas J, Tuimala J, Paulin L, et al. Microbial community analysis reveals high level phylogenetic alterations in the overall gastrointestinal microbiota of diarrhoea-predominant irritable bowel syndrome sufferers. BMC Gastroenterol. 2009;9:95

26. Pimentel M, Chow EJ, Lin HC. Eradication of small intestinal bacterial overgrowth reduces symptoms of irritable bowel syndrome. Am J Gastroenterol. 2000;95:3503-3506.

27. Ghoshal UC, Shukla R, Ghoshal U. Small Intestinal Bacterial Overgrowth and Irritable Bowel Syndrome: A Bridge between Functional Organic Dichotomy. Gut Liver. 2017;11:196-208.

28. Lin HC. Small intestinal bacterial overgrowth: a framework for understanding irritable bowel syndrome. JAMA. 2004;292:852-858

29. VerdúEF, Bercík P, Bergonzelli GE, Huang XX, Blennerhasset $\mathrm{P}$, Rochat $\mathrm{F}$, et al. Lactobacillus paracasei normalizes muscle hypercontractility in a murine model of postinfective gut dysfunction. Gastroenterology. 2004;127:826-837.

30. Verdú EF, Bercik P, Verma-Gandhu M, Huang XX, Blennerhassett $\mathrm{P}$, Jackson W, et al. Specific probiotic therapy attenuates antibiotic induced visceral hypersensitivity in mice. Gut. 2006;55:182-190. 